\section{Cahiers de Narratologie}

Analyse et théorie narratives

$7 \mid 1996$

Mélanges espace \& temps

\title{
Petit précis de narratologie
}

\section{Gérard Lavergne}

\section{OpenEdition}

Journals

Édition électronique

URL : https://journals.openedition.org/narratologie/11769

DOI : 10.4000/narratologie.11769

ISSN : 1765-307X

Éditeur

LIRCES

Édition imprimée

Date de publication : 1 janvier 1996

Pagination : 23-33

ISSN : 0993-8516

\section{Référence électronique}

Gérard Lavergne, «Petit précis de narratologie ». Cahiers de Narratologie [En ligne], 7| 1996, mis en ligne le 23 février 2021, consulté le 05 mai 2021. URL : http://journals.openedition.org/narratologie/ 11769 ; DOI : https://doi.org/10.4000/narratologie.11769

Ce document a été généré automatiquement le 5 mai 2021.

Article L.111-1 du Code de la propriété intellectuelle. 


\title{
Petit précis de narratologie
}

\author{
Gérard Lavergne
}

1 Ce travail m'a été demandé pour aider les étudiants et agrégatifs du Département d'études Hispaniques et Hispano Américaines à résoudre les problèmes de narratologie que posent les ouvrages proposés aux programmes de l'agrégation et du Capes 1996. En particulier Kaila et Dimna (Les fables de Bidpay); Beatus Ille de Antonio Muñoz Molina (1986)

2 Un texte est un tout (fini) achevé, structuré, qui se compose de signes linguistiques ou iconographiques (un dépliant, une affiche, sont des textes).

3 Un texte narratif sera celui où un agent raconte une histoire (=fiction= fable) : ce sera l'ensemble des signes linguistiques porteurs de cette histoire.

4 L'histoire est une fiction, une fable, présentée d'une certaine façon.

5 Une fiction est une série (d'éléments) d'événements fictionnels et chronologiques reliés logiquement et chronologiquement entre eux et dont les acteurs sont la cause ou en supportent les conséquences.

6 Les acteurs sont les agents, pas nécessairement humains, qui mènent l'action. Ne pas confondre 3 concepts différents : personnage, acteur, actant.

7 La narration c'est, portée par le texte narratif, la présentation dans le discours de la fiction et de la manière de la conter.

8 Un événement est la transition d'un état à un autre, d'une situation à une autre, au cours d'un temps.

9 Agir : causer ou subir un événement

10 Affirmer qu'un texte narratif est celui où l'on raconte une histoire implique que le texte n'est pas l'histoire : plusieurs textes peuvent évoquer la bataille de Waterloo, plusieurs textes peuvent évoquer le conte de Cendrillon.

11 Plusieurs textes peuvent donner plusieurs narrateurs de l'histoire, de la fiction de Cendrillon, du petit chaperon rouge. On peut avoir un texte littéraire, un texte filmique, une bande dessinée... 
12 Auteur : Muñoz Molina, né le.., à..., académicien; Balzac, Faulkner... ce sont des êtres de chair et de sang.

13 Scripteur : A. Muñoz Molina quand il écrit un roman; car à ce moment n'entre en jeu qu'une part de sa personnalité d'auteur. Il choisit, prend, élimine, imagine, complète tel ou tel détail, construit, compose le temps, l'espace, ses personnages, la diégèse. C'est donc l'auteur en état de grâce pour créer telle fiction (récit fictionnel). C'est un concept qui ressort à la fois du réel et du fictif.

14 Le narrateur...narre; car il sait (gnarus). Mais il ne raconte qu'en fonction des qualifications qui lui sont attribuées, de sa présence ou de son absence dans la fiction, de son rôle (actif ou non actif) dans la fiction.

- Il peut être le personnage principal (roman autobiographique, picaresque) il peut se confondre avec l'auteur.

- Il peut être un ou plusieurs personnages secondaires (vision plurielle du même événement), difficile de dire là où est l'auteur.

- Il peut n'être personne : mais on peut alors considérer que nous avons là un narrateur qui n'est pas dans la fiction, mais qui parle à la troisième personne.

Remarques: Il existe plusieurs types de voix narratives, unique (le narrateur), ou plurielle (penser aux murmures d'un village, aux médisances ou le «on dit»: « dijeron »)

De plus, toute narration n'est pas obligatoirement orale (photographie, cinéma muet, portrait, document ; monument, pièces à conviction, traces....)

\section{Statut du narrateur il est double.}

a) il est absent (ou il n'est plus présent) dans la fiction.

absent $=$ extradiégétique

présent = intradiégétique .

b) il est personnage : il prend ou il a pris part au récit (il agit ou il a agi).

s'il agit lui-même, s'il s'agit de lui : homo ou autodiégétique ;

s'il n'agit pas dans ce qu'il raconte : hétérodiégétique.

On a donc une fonction narrative :

a) 1 : Le narrateur situé au $1^{\circ}$ degré, donc situé à l'extérieur de l'histoire qu'il raconte, Clarín, Mendoza, Homère, César, = narrateur extradiégétique.

2 : Le narrateur au second degré qui apparaît dans l'histoire racontée au $1^{\text {er }}$ degré, Shéhérazade dans les Mille et une nuits = narrateur intradiégétique.

b) $1:$ Le narrateur est lui-même personnage de l'histoire qu'il raconte : le Picaro, Des Grieux

= narrateur homodiégétique.

2 : Le narrateur n'est pas un personnage de l'histoire qu'il raconte :

Shéhérazade : elle raconte l'histoire d'Aladin

= narrateur hétérodiégétique.

Donc on peut avoir :

- un N extradiégétique-hétérodiégétique : Martin Santos 
- un $\mathrm{N}$ extradiégétique-homodiégétique: Le Pícaro, Jacinto, César et la guerre des gaules, Solana raconte son histoire à Minaya

- un N intradiégétique-hétérodiégétique : Shéhérazade. Avec E. Pardo Bazan in Los Pazos de Ulloa, les narrateurs au second degré (intra), racontent des histoires auxquelles ils ne participent pas. (Les chasseurs)

- un $\mathrm{N}$ intradiégétique-autodiégétique: un narrateur au second degré raconte une histoire qui le concerne : un policier raconte son enquête à un collègue dans un roman policier à la $3^{e}$ personne. Rodrigue raconte (Le Cid) son arrivée au port et la défaite des Mores. Ulysse (chants IX-XII) raconte sa propre histoire.

Remarque : le Narrateur en Abyme est le narrateur $1^{\mathrm{e}}$ qui raconte qu'un narrateur $2^{\mathrm{e}}$ lui a raconté qu'il a appris d'un narrateur $3^{\circ} \ldots$

Un exempte : dans Beatus Ille

Un narrateur extra-hétérodiégétique (Muñoz Molina l'auteur) raconte à la $3^{\text {ème }}$ personne l'histoire d'un étudiant, Minaya, qui fuit Madrid et la répression politique, pour se rendre à Mágina chez son oncle, sous le prétexte de rédiger une thèse qui portera sur la biographie d'un poète présumé de la génération de 27 décédé, Jacinto Solana, ami de cet oncle. Il cherche les éléments pour faire ce travail. Jacinto Solana, qui en fait n'est pas mort mais s'est réfugié dans la solitude (Beatus Ille). apprend par Inès sa nièce, les intentions de Minaya. Il décide de donner indirectement par sa nièce à ce dernier les informations nécessaires à la réalisation de cette biographie.

Nous avons donc le personnage Minaya, narrateur intradiégétique dans le Beatus Ille de Muñoz Molina et Hétérodiégétique de la biographie qu'il rédige, qui change de statut et devient de fait le personnage narrateur 2d.. intradiégétique manipulé par Jacinto Solana qui, par son intermédiaire, se révèle le narrateur $1^{\mathrm{e}}$ extra-homodiégétique (car s'il s'agit de lui il n'est plus présent) de l'œuvre, rédigée par Minaya auteur de la biographie, à qui il fait rédiger ainsi le Beatus Ille qu'il a toujours rêvé d'écrire.

41 Pour ce faire, Minaya utilise les documents soit-disant trouvés par lui, et quand il n'a plus de documents, il change de statut : de chercheur il devient créateur de la fiction. Il invente en effet les éléments qui lui manquent et il mythifie son personnage, un poète de la génération de 1927.

Pour résumer nous avons ici un narrateur à la $3^{\text {ème }}$ personne qui raconte Minaya qui raconte " un » Solana, que le même Solana a raconté à Minaya. On pourrait parler ici de narrateur non seulement en abyme mais circulaire.

43 Le narrataire : celui à qui, dans la fiction, le narrateur raconte son histoire ou une histoire. Jacinto Solana narrateur (III ${ }^{\text {ème }}$ partie) raconte à Minaya (narrataire) l'histoire d'Inès et la sienne. Shéhérazade racontée par un narrateur extradiégétique devient narrateur intradiégétique et a pour narrataire le roi qu'elle séduit.

Le destinataire : Celui à qui est adressé le texte en dehors de la fiction. Il peut être multiforme : Amis lecteurs.... ou ciblé : un auteur à un autre, un poète à sa fille...

Dans le roman de Cela La Muerte de Pascual Duarte, Pascual Duarte destine son texte, sa confession, à « Monsieur Joaquin Barrera Lopez Merida » en tant qu'ami de celui qu'il a assassiné.

Le récit est le fait de deux éléments :

- La fiction : ce que l'on raconte : le signifié 
48

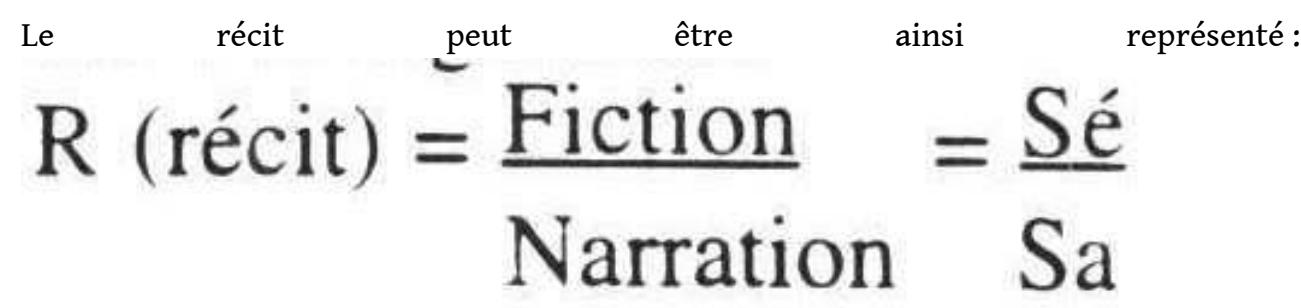

D'après Genette (Figure III p. 238) : Tout événement raconté dans un récit est à un niveau diégétique immédiatement supérieur à celui où se situe l'acte narratif producteur.

Il s'agit ici du récit dans le récit.

Nous parlerons pour la plus grande clarté du propos, d'un thème principal dans une fiction que nous appellerons le récit premier; ce dernier sera éclairé par des minirécits (récits seconds), qui eux- mêmes pourront être explicités par d'autres récits $3^{\mathrm{e}} \ldots$

51 Dans le même but de clarté nous pensons logique de réserver l'expression " métatexte " à un texte porteur d'un métadiscours, d'un discours sur la langue, ou sur le discours luimême; dans Beatus Ille le narrateur-auteur «Jacinto Solana-Muñoz Molina » dit cf. p. 277 «Qu'une histoire soit vraie ou fausse, cela ne compte pas mais ce qui compte c'est qu'on sache la raconter ».

C'est un métatexte qui a en fait pour base les catégories de la rhétorique : l'invention, la disposition, l'élocution.

Le récit spéculaire est dans un récit premier un récit second qui reprend le thème du récit premier: dans Beatus Ille nous avons l'histoire d'un personnage qui écrit par l'intermédiaire d'un autre personnage un roman qu'il a toujours voulu écrire, intitulé Beatus Ille.

54 - Se rappeler le tableau de Jan Van Eyck « Arnolfini et sa femme (1434).

55 - Se rappeler également les Ménines de Velacquez (1656) où les personnages du $\mathrm{I}^{\mathrm{e}}$ plan regardent les spectateurs en dehors de la toile (le Roi et la Reine) que l'on voit se refléter dans un miroir tout comme dans Beatus Ille p. 29. Mariana regarde derrière le photographe les spectateurs. Le peintre Orlando compose son tableau en évoquant également Vélazquez et les Ménines p. 193.

56 Les actants Greimas a regroupé les personnages dans des catégories qui rassemblent non des acteurs, choses... ni des personnages (êtres), mais un ensemble de caractères qui détermine une manière d'être et de faire, " une force qui agit ", au cours de la Quête que représente le récit.

57 Il situe les actants au nombre de 6 selon le schéma actantiel suivant :

\section{destinateur $\rightarrow$ objet $\rightarrow$ destinataire $\uparrow$ adjuvant $\rightarrow$ sujet $\rightarrow$ opposant.}

Attention: personnage, acteur, actant, sont 3 concepts différents et non interchangeables. 
Minaya est personnage et acteur.

Les miroirs, les photographies, une hache sont des acteurs.

Le traître est un opposant., l'amour, la jeunesse sont des adjuvants ou des opposants... tout dépend de la quête.

Le personnage.

On peut partir de la définition de $\mathrm{Ph}$. Hamon qui considère le personnage comme « un morphème doublement articulé manifesté par un signifiant discontinu (un certain nombre de marques) renvoyant à un signifié discontinu (le «sens» ou la valeur du personnage)»

Poétique du récit «Statut semi-logique du personnage » Col. points n 78, p. 124 ;

On considérera du personnage

- le statut qualificatif (comment il est campé, les éléments qui le composent)

- le statut actantiel (son rôle dans la fiction)

- le statut narratif (son rôle dans la production du récit)

- le statut idéologique (son sens ou signifié)

Pour ce qui est de son être (qualificatif) il faut considérer :

Les indices (faits, allusions, signifiés implicites)

les informants (époque, atmosphère, lieu, durée)

ensemble d'éléments qui permettent d'étudier le faire, l'être et le paraitre (vérifier si les indices et les informants sont toujours exacts...)

Penser aux définitions de E. M. Forster (aspects of the novel).

- Le personnage rond (il évolue, il peut surprendre dans son comportement)

- Le personnage plat (sans surprise)

on peut évoquer les personnages \pm référentiels.

N. Dans beatus Ille il nous semble que, mis à part Minaya, nous n'avons que des personnages plats- pour ne pas dire des rôles-: le bon, le méchant, le traitre, le mythe, la victime innocente, l'hypocrite, le juste...

\section{Les modes}

Diégèses : description et narration. C'est l'histoire, le récit, la parole du narrateur.

Mimesis : Représentation, imitation, discours direct, drame = parole du personnage à qui l'auteur cède la parole. C'est parfois la réflexion, l'intrusion d'auteur : comparaison, réflexion générale (métalepse). Beatus Ille p. 106.

Attention, on peut trouver la narration dans le drame, et la représentation dans le récit non dialogué.

\section{Le temps}

Dans tout récit il y a :

a) le temps de la fiction (de la "chose» annoncée) c'est à dire de la chaîne événementielle supposée réelle de la fiction. Ce sera le temps de l'énoncé.

b) le temps de la narration : la chaîne événementielle telle qu'elle apparaît dans le récit. Ce sera le temps de L'Énonciation.

c) le temps narré sera donné par la distorsion entre les deux chaînes. 

présent. Cf. par exemple Beatus Ille ch. 5, p. 44.

\section{La disposition dans un récit}

Dans un récit on peut ne trouver qu'une histoire. Mais il existe des récits plus complexes qui enserrent plusieurs histoires. Ces histoires seront reliées entre elles selon les procédés de :

110 - l'enchâssement : les Mille et une nuit, Kalila et Dimna, le Décaméron de Boccace.

111 Dans un roman le narrateur extrahétérodiégétique raconte une réunion où un ou plusieurs personnages racontent une histoire. Ils sont ou deviennent narrateur intrahétérodiégétique d'un récit premier enchâssant différents récits seconds. 
112 Note. Attention à la métalepse : c'est l'intervention (l'intrusion) du narrateur, de l'auteur, du personnage, qui s'adresse au lecteur ou inversement c'est l'intervention enchâssée certes, qui fait du discours, mais ne crée pas de fiction. état de : Intérêts divers : intéresser le lecteur, créer de l'étrange, divertir...

- l'alternance : consisté à raconter simultanément plusieurs histoires ou plusieurs tranches de vie : Los Otros de Luis Romero, le genre épistolaire. Les Liaisons dangereuses de Choderlos de Laclos

- l'enchaînement consiste à juxtaposer différentes histoires, avec entre elles des ressemblances dans le thème ou la structure

Las Novelas amorosas y ejemplares de Maria de Zayas y Sotomayor 1637

La Noria de Luis Romero, séries de tranches de vie coordonnées par un thème (la vie de Barcelone par exemple).

C'est le procédé utilisé dans le film La Ronde de Max Ophuls (1950) Ronde des aventures ou mésaventures amoureuses: Une jeune fille est amoureuse d'un jeune homme qui l'abandonne et rencontre une autre jeune fille qui l'abandonne et choisit un autre homme qui...

\section{Le point de vue La focalisation}

Le problème est de savoir :

1 qui voit la scène, les personnages, le paysage X... ?

2 qui parle et narre ce que voit celui qui voit la scène... ?

La focalisation. Il ne s'agit pas de réduire le terme de focalisation à une idée de vision seule. Il faut l'étendre à l'idée de perception et de perspective.

$1^{\mathrm{er}}$ problème : qui, dans la fiction, perçoit un phénomène ?, qui fixe ce phénomène ?, réfléchit, l'étudié, parle? Qui pense et dit des choses? donc qui focalise sur le phénomène $\mathrm{X}$ en question qu'il voit en fonction d'une perspective, la sienne ? Ce sera le personnage idéalisateur : le focalisateur focalise le focalisé.

$2^{\text {ème }}$ problème : qui narre ce que voit, perçoit, fixe le personnage focalisateur? Ce sera le narrateur.

le point de vue :

Le narrateur va narrer en fonction du pouvoir de connaissance que lui a accordé le scripteur. C'est le problème de la vision, du point de vue. Selon ce pouvoir il sera en

- Tout savoir : omniscient, il connaît le passé, l'avenir, les pensées, les personnages

C'est la vision par derrière (Pouillon)

appelée focalisation zéro par Genette

- Ne savoir que ce que voit et sait son personnage. Il connaît par et avec le personnage.

C'est la vision avec (Pouillon)

la focalisation interne (Genette)

- Il ne connaît et ne sait que ce que peut connaitre et voir un spectateur anonyme observant la scène comme lui, de l'extérieur ; (c'est l'œil de la caméra).

C'est la vision du dehors (Pouillon)

la focalisation externe (Genette) 


\section{Récit et Discours}

141 Le narrateur narre, rapporte, ce que dit, ce que pense le personnage qu'il focalise : c'est le récit. fiction, hétérodiégétique, et celle d'un narrateur présent dans la fiction, homodiégétique.

\section{Dans le récit}

144 - On ne sait qui parle, on ne sait pas quelle est l'origine de l'énonciation. C'est un récit fait à la troisième personne par un narrateur hétérodiégétique qui parle des personnages. C'est le système dit de la non-personne (il, ils, les indéfinis, les noms...)

145 - Il a pour temps de base le passé simple, avec l'imparfait et le plus-que-parfait.

146 - les repérages, les déictiques temporels seront du domaine de l'énoncé : le 18 juillet, alors, ce mois- là, 3 jours après le lendemain, trois jours plus tôt...

147 Les déictiques indiqueront l'éloignement : là-bas, voilà...

\section{Dans le discours :}

148 - L'énonciation (le locuteur) est présente avec le système dit de la première personne, avec les pronoms qui renvoient aux personnages de l'acte d'énonciation (je, me, moi, nous vous)

149 - Le temps de base est le présent, avec le futur, le passé composé et l'imparfait et le plus-que-parfait.

150 On peut noter que l'imparfait "un présent dans le passé ", de par sa nature peut participer à l'instauration d'une durée plus ou moins vague, à une stagnation, une sorte d'arrêt sur l'image où les événements peuvent perdre de leur vigueur et se fondre dans une incertitude prononcée, dans un flou peut-être annonciateur d'un passage de L'Histoire au Mythe (Érase que se era... il était une fois)

151 - Les repérages temporels se feront par rapport au présent et par rapport à la $1^{\text {ère }}$ personne : aujourd'hui, demain, hier, dans trois jours, il y a deux mois

152 - Les déictiques indiqueront la proximité : ici, voici, celui-ci, ce lieu ci. Il est organisé par rapport au sujet parlant. 
153 Le problème dans la relation Récit-Discours est celui du plus ou moins grand engagement du narrateur vis-à-vis de son personnage, dans la façon qu'il a de transmettre au lecteur, de rapporter le « dit » ou le «pensé » de ce personnage. Feint-il de lui donner la parole? Se contente-t-il de raconter ou de résumer ce qu'il a dit sans répéter le discours vraiment prononcé ? De son attitude découlent les genres différents de discours.

\section{Le discours rapporté}

154 - Le discours direct. Le Narrateur (N) annonce ce qui va être dit par le personnage (P). Il est responsable du verbe déclaratif (dire, annoncer) et de la modalité choisie (clamer, hurler, menacer, blâmer), mais il ne prend aucune responsabilité dans ce qui est énoncé. Il donne l'illusion que ce n'est pas lui qui parle.

155 En évoquant Inès dans Beatus Ille, $\mathrm{N}$ pourrait dire :

156 La jeune femme affirma (ajouta, s'écria, dit, hurla)(l) « Je ne veux pas m'en aller »(2).

157 Annoncé par N (1), P(2) cite son propre discours. Il est en quelque sorte son propre narrateur. Il parle à la $1^{\text {ère }}$ personne. La distance entre $\mathrm{N}$ et $\mathrm{P}$ (Narrateur et personnage énonciateur) est égale à zéro.

Dans ce discours le narrateur à la troisième personne est totalement absent.

159 - Le discours indirect : Il marque une reprise en charge du discours par $\mathrm{N}$ à la troisième personne : «La jeune femme affirma qu'elle ne voulait pas s'en aller» ou bien la jeune femme affirma ne pas vouloir s'en aller ».

$160 \mathrm{~N}$ apparait dans le verbe déclaratif (annonça) de la principale, et les parole de $\mathrm{P}$ se trouvent dans la subordonnée complétive ou dans l'infinitif. La proposition est régie par le verbe de la principale et les paroles sont rapportées sous la responsabilité du narrateur.

161 - Le discours indirect libre: forme intermédiaire entre les deux précédentes sans subordination, comme le discours direct, il est soumis à l'énonciation du narrateur : " elle ne voulait pas s'en aller »

162 Ce discours garde le jeu syntaxique induit par le verbe principal d'un discours indirect, mais il prend la valeur expressive de ce discours. Cependant on peut parfois se demander si ce sont les propos du personnage ou le récit du narrateur que nous lisons. Dans le cas du discours indirect et du discours indirect libre (Genette parle alors de discours transposé) on peut dire que la présence du narrateur est sensible mais pas prépondérante et que la distance entre $\mathrm{N}$ et $\mathrm{P}$ est minimale.

163 - Le discours narrativisé dont parle Genette: ici la distance entre $\mathrm{N}$ et $\mathrm{P}$ sera maximale $(D=\infty)$. C'est le discours le plus distant. Le narrateur fait du discours un événement, un élément de son récit comme un autre: «la jeune femme affirma son refus ".

Ici on annonce un événement sans tenir compte du discours énoncé.

165 - Le monologue intérieur (le discours immédiat de Genette).

166 Dans le rapport Récit-Discours nous avons vu un amalgame, un rapprochement des statuts Narrateur-Personnage plus ou moins distant, une passation du pouvoir de parler du narrateur au personnage. Nous avons avec le monologue intérieur une 
émancipation d'emblée totale de l'instance narrative: le narrateur extradiégétique disparaît. La distance N-P est ici égale à zéro.

Le lecteur est placé dans l'esprit du personnage dont la pensée, le flux de la conscience, s'écoule au style direct : à la $1^{\text {ère }}$ personne, avec le présent pour temps de base.

On peut trouver cependant un discours à la $3^{\text {ème }}$ personne -penser au style indirect libre-, discours qui, réécrit à la $1^{\text {ère }}$ personne, (dans la mesure où « cette opération n'entraîne aucune altération du discours que le changement même des pronoms grammaticaux» -Barthes, Communication 8-) se révèle être les pensées d'un personnage.

169 Pour conclure ce résumé, nous dirons que l'approche narratologique doit conduire à une plus profonde appréhension sémiotique et téléologique de l'œuvre, du sens de l'œuvre et de sa finalité.

170 Si nous nous en référons à Beatus Ille, il est évident que nous pouvons voir là, non seulement une excellente application de techniques narratives, une sorte d'ars escribendi, mais aussi une virulente remise en cause de certains mythes très vivaces des années 30, autant celui de l'intellectuel des années 30 au service du peuple, que celui d'un temps idéal promis pour un avenir proche. En d'autres termes, nous dirons que ce roman est un excellent exemple de roman post-moderne.

\section{BIBLIOGRAPHIE}

GENETTE Gérard : (1972), Figures III, Paris, Seuil, Col. Poétique, 286 p.

GOLDENSTEIN J. P. : (1989), Pour lire le roman, Bruxelles, Paris, Deboek Duculot.

HAMON Philippe : (1977), « Pour un statut sémiologique du personnage ». Poétique du récit, Paris, Seuil, Col. Points $n^{\circ} 78$, p. 115-180.

POUILLON Jean : (1946), Temps et Roman, Paris Gallimard.

REUTER Yves : (1991), Introduction à l'analyse du roman, Paris, Bordas, $165 \mathrm{p}$.

Analyse structurale du récit, Communications 8, Paris, Seuil, 1966, 172 p.

\section{AUTEUR}

\section{GÉRARD LAVERGNE}

Université de Nice-Sophia Antipolis 